\title{
Existence of Stark Ladder Resonances
}

\author{
Jim Agler ${ }^{1,2}$ and Richard Froese \\ Department of Mathematics, California Institute of Technology, Pasadena, CA 91125, USA
}

\begin{abstract}
We show that resonances, in the translation analytic sense of Herbst and Howland, exist for the one dimensional Stark Hamiltonian, $-d^{2} / d x^{2}+q(x)$ $+\varepsilon x$, with $q(x)$ a trigonometric polynomial, provided $\varepsilon$ is sufficiently large.
\end{abstract}

\section{Introduction}

The problem of describing the motion of a particle in a one dimensional periodic solid pervaded by a uniform electric field has received considerable attention in the physics literature (see, e.g. $[1,10,11]$ ). Controversy has centered about the existence of so-called Wannier states, or Stark ladder resonances, which were described by Wannier in [9]. The purpose of this paper is to prove that for periodic potentials given by trigonometric polynomials resonances in the translation analytic sense of Herbst and Howland [5] exist for large values of the electric field.

The Hamiltonian for the system in question is

$$
H(\varepsilon)=-\frac{d^{2}}{d x^{2}}+\varepsilon x+q(x)
$$

acting in $L^{2}(\mathbb{R})$, where $q(x)=\sum_{n} c_{n} e^{i n x}$ is a real valued trigonometric polynomial. Here $\varepsilon$ is the strength of the electric field. It is known that for $\varepsilon \neq 0, \sigma(H(\varepsilon))=\mathbb{R}$ and is purely absolutely continuous $[3,4]$. To describe translation analyticity we begin with the unitary group of translations

$$
(T(a) f)(x)=f(x+a)
$$

for $a \in \mathbb{R}$ and note that

$$
T(-a) H(\varepsilon) T(a)=-\frac{d^{2}}{d x^{2}}+\varepsilon x+q(x-a)-\varepsilon a .
$$

Since $q(x)$ has an analytic extension to complex $x$ we can define the complex

1 Permanent Address: UCSD - San Diego, CA 92093, USA

2 Work partially supported by NSF grant DMS- 8409630 
translated Hamiltonian

$$
H(\varepsilon, \alpha)=-\frac{d^{2}}{d x^{2}}+\varepsilon x+q(x-i \alpha)-i \varepsilon \alpha
$$

for $\alpha \in \mathbb{R}$. In the case at hand, since $q(x-i \alpha)$ is bounded in $x, H(\varepsilon, \alpha)$ can be defined as a closed operator on $\mathscr{D}\left(p^{2}+\varepsilon x\right)$. Herbst and Howland prove (for a much larger class of $q)$ that $\sigma_{\text {ess }}(H(\varepsilon, \alpha)) \subseteq \mathbb{R}-i \varepsilon \alpha$ and $\sigma_{\text {disc }}(H(\varepsilon, \alpha)) \subseteq$ $\{z:-i \varepsilon \alpha \leqq \operatorname{Im} z \leqq 0\}$. The eigenvalues in $\sigma_{\text {disc }}(H(\varepsilon, \alpha)) \backslash(\mathbb{R}-i \varepsilon \alpha)$ are the resonances. They don't move as $\alpha$ changes once they have been "uncovered" by the line containing essential spectrum. They can also be shown to be poles of suitable matrix elements of the resolvent of $H(\varepsilon)$ continued into the lower half plane. What we prove is that for large values of $\varepsilon, H(\varepsilon, \alpha)$ indeed has eigenvalues not on the line $\mathbb{R}-i \varepsilon \alpha$, for some value of $\alpha$. Since there is the unitary equivalence

$$
T(-2 \pi n) H(\varepsilon, \alpha) T(2 \pi n)=H(\alpha, \varepsilon)-2 \pi \varepsilon n
$$

for $n \in \mathbb{Z}$, the existence of one resonance implies the existence of a whole sequence, the so-called Stark ladder.

We briefly mention some of the folklore surrounding this problem. The spectrum of $H(0)$ has the familiar band structure. Let $P_{n}$ project onto the $n^{\text {th }}$ band subspace of $H(0)$. Then for $\varepsilon \neq 0$ the operators $P_{n} H(\varepsilon) P_{n}$ and thus $\sum_{n} P_{n} H(\varepsilon) P_{n}$ have discrete spectrum. It is thought these bound states should persist as resonances when the off-diagonal parts $P_{n} H(\varepsilon) P_{m}, n \neq m$ are added to $\sum_{n} P_{n} H(\varepsilon) P_{n}$ to give $H(\varepsilon)$. Previous work has centered about proving that the off-diagonal parts are small as $\varepsilon \downarrow 0$ $[2,7]$. So far this approach has not led to a proof of the existence of Stark resonances.

We make no use of the above intuition. Instead we introduce an operator inspired by the Birman-Schwinger operators common in Schrödinger operator theory. The fact that this operator is compact follows from work in [4].

Our results are summarized in the following theorem.

Theorem 1. Let $q(x)$ be the trigonometric polynomial given by

$$
q(x)=\sum_{n=-N}^{N} c_{n} e^{i n x}, c_{n} \in \mathbb{C},
$$

where $c_{0}=0$, and $c_{n} \neq 0$ for at least one $n>0$. Let $p=-i d / d x$. For $\varepsilon>0$ and $\alpha \in \mathbb{R}$ define the closed operator

$$
H(\varepsilon, \alpha)=p^{2}+\varepsilon x+q(x-i \alpha)-i \varepsilon \alpha
$$

acting in $L^{2}(\mathbb{P})$ with domain $\mathscr{D}(H(\varepsilon, \alpha))=\mathscr{D}\left(p^{2}+\varepsilon x\right)$. Then there exists $\varepsilon_{0}>0$ such that for every $\varepsilon>\varepsilon_{0}$ there exists $\alpha \in \mathbb{R}$ such that $H(\alpha, \varepsilon)$ has an eigenvalue not contained in the line $\mathbb{R}-i \varepsilon \alpha$.

In this theorem $q(x)$ need not be real on the real axis. However if it happens to be real on the real axis, then the results of Herbst and Howland apply and Theorem 1 asserts the existence of Stark resonances. We suspect, but cannot prove, that in fact $\varepsilon_{0}$ may be taken to be zero in Theorem 1 . 


\section{A Birman-Schwinger Type Operator}

Let the symbol $[\cdot]^{1 / 2}$ denote a branch of the square root, fixed throughout the rest of the paper, which is analytic in the lower half plane. Throughout this paper $\varepsilon>0$ and $\operatorname{Im} \mu>0$. Define

$$
M(\varepsilon, \mu)=\left[p^{2}+\varepsilon x-\mu\right]^{-1 / 2},
$$

where $p=-i d / d x$. Let

$$
S(\varepsilon, \mu, z)=M(\varepsilon, \mu) \sum_{n=-N}^{N} c_{n} z^{n} e^{i n x} \quad M(\varepsilon, \mu),
$$

where the $c_{n}$ are the coefficients in $q(x)$ (see Theorem 1). We have the following analogue of the Birman-Schwinger principle.

Lemma 1. Fix $\alpha \in \mathbb{R}$ and $\varepsilon>0$. Let $S(\varepsilon, \mu, z)$ be as above and let $H(\varepsilon, \alpha)$ be given by (1.2). Then $H(\varepsilon, \alpha)$ has an eigenvalue at $\lambda$ iff $S(\varepsilon, \mu, z)$ has an eigenvalue at -1 , with

$$
z=e^{\alpha+i a}, \quad \mu=\lambda+i \varepsilon \alpha+\varepsilon a
$$

for some choice of $a \in \mathbb{R}$.

Proof. Suppose $H(\varepsilon, \alpha)$ has eigenvalue $\lambda$. This means there exists $\psi \in \mathscr{D}(H(\varepsilon, \alpha))$ such that

$$
H(\varepsilon, \alpha) \psi=\lambda \psi
$$

This equation can be rewritten as

$$
M^{-2} \psi=-q(x-i \alpha) \psi
$$

where $M=M(\varepsilon, \lambda+i \varepsilon \alpha)$. Here we use that $\mathscr{D}(H(\varepsilon, \alpha))=\mathscr{D}\left(M^{-2}(\varepsilon, \lambda+i \varepsilon \alpha)\right)$. Using (2.4) it is easy to see that $\phi=M^{-1} \psi$ satisfies

$$
S\left(\varepsilon, \lambda+i \varepsilon \alpha, e^{\alpha}\right) \phi=-\phi .
$$

Conversely, if (2.5) holds for some $\phi \in L^{2}$, i.e. if $S\left(\varepsilon, \lambda+i \varepsilon \alpha, e^{\alpha}\right)$ has eigenvalue -1 , then it follows that $\psi=M \phi$ is in $\mathscr{D}(H(\varepsilon, \alpha))$ and satisfies (2.3).

For any $a \in \mathbb{R}$ we have the unitary equivalence

$$
T(-a) S(\varepsilon, \mu, z) T(a)=S\left(\varepsilon, \mu+\varepsilon a, e^{i a} z\right),
$$

where $T(a)$ is given by (1.1). Thus $S\left(\varepsilon, \lambda+i \varepsilon \alpha, e^{\alpha}\right)$ has eigenvalue -1 iff $S\left(\varepsilon, \lambda+i \varepsilon \alpha+\varepsilon a, e^{\alpha+i a}\right)$ has eigenvalue -1 for some $a \in \mathbb{R}$. The proof is complete.

Given Lemma 1, the following theorem implies Theorem 1.

Theorem 2. There exists $\mu_{0}$ and $\varepsilon_{0}$ with $\operatorname{Im} \mu_{0}>0$ and $\varepsilon_{0}>0$ such that for every $\varepsilon>\varepsilon_{0}, S\left(\varepsilon, \mu_{0}, z\right)$ has eigenvalue - 1 for some value of $z$.

We conclude this section with an outline of the proof of Theorem 2. We will show that $S(\varepsilon, \mu, z) \in \mathscr{I}_{4}$, the fourth trace ideal class (see [8] for definitions and properties of trace ideals). It then follows that $S(\varepsilon, \mu, z)$ has eigenvalue -1 iff $\operatorname{det}_{4}(1+S(\varepsilon, \mu, z))$ vanishes, where $\operatorname{det}_{4}$ is the regularized determinant. We will show that for some fixed $\mu_{0}$ with $\operatorname{Im} \mu_{0}>0, \ln \operatorname{det}_{4}\left(1+S\left(\varepsilon, \mu_{0}, z\right)\right)$ is analytic in $z$ 
for $|z|<1$. Thus for $|z|<1$

$$
\ln \operatorname{det}_{4}\left(1+S\left(\varepsilon, \mu_{0}, z\right)\right)=\sum_{\ell=0}^{\infty} f_{\ell}(\varepsilon) z^{\ell} .
$$

Let $\mathscr{E}$ denote the set of $\varepsilon$ for which $S\left(\varepsilon, \mu_{0}, z\right)$ never has eigenvalue -1 , as $z$ ranges through $\mathbb{C} \backslash\{0\}$ If $\varepsilon \in \mathscr{E}$ then $\operatorname{det}_{4}(1+S(\varepsilon, \mu, z))$ never vanishes, so the left side of (2.6) is entire in $z$ and it will be shown that in that case that case the left side of (2.6) is in fact a polynomial of degree at most $4 N_{1}$. Thus, $\mathscr{E}$ must lie in the zero set of $f_{\ell}$ for all $\ell>4 N_{1}$. A calculation showing $f_{5 N_{1}}$ does not vanish for large $\varepsilon$ will complete the proof.

\section{Proof of Theorem 2}

Instead of dealing with $S(\varepsilon, \mu, z)$ directly we work with an operator unitarily equivalent to it. For $\varepsilon>0$ let $U(\varepsilon)=\exp \left(i p^{3} / 3 \varepsilon\right)$. This operator is common in the theory of Stark Hamiltonians as it has the property that

$$
U(\varepsilon)^{*}\left(p^{2}+\varepsilon x\right) U(\varepsilon)=\varepsilon x .
$$

Define

$$
R(\varepsilon, \mu, z)=U(\varepsilon)^{*} S(\varepsilon, \mu, z) U(\varepsilon)
$$

Using the identities

$$
\begin{gathered}
U(\varepsilon)^{*} M(\varepsilon, \mu) U(\varepsilon)=[\varepsilon x-\mu]^{-1 / 2} \\
e^{-i \alpha x} f(p) e^{i \alpha x}=f(p+\alpha), \quad \text { for } f \text { bounded and measurable, }
\end{gathered}
$$

and

$$
\begin{aligned}
U(\varepsilon)^{*} e^{i n x} U(\varepsilon) & =e^{i(n / 2) x} \exp \left(-i\left(p+\frac{n}{2}\right)^{3} / 3 \varepsilon\right) \exp \left(i\left(p-\frac{n}{2}\right)^{3} / 3 \varepsilon\right) e^{i(n / 2) x} \\
& =\exp \left(-i n^{3} / 12 \varepsilon\right) e^{i(n / 2) x} \exp \left(-\frac{i n}{\varepsilon} p^{2}\right) e^{i(n / 2) x}
\end{aligned}
$$

it is easy to see that

$$
R(\varepsilon, \mu, z)=\sum_{n=-N}^{N} c_{n} z^{n} K_{n}(\varepsilon, \mu)
$$

where

$$
K_{n}(\varepsilon, \mu)=e^{-i n^{3} / 12 \varepsilon}[\varepsilon x-\mu]^{-1 / 2} e^{i(n / 2) x} \exp \left(i-\frac{n}{\varepsilon} p^{2}\right) e^{i(n / 2) x}[\varepsilon x-\mu]^{-1 / 2} .
$$

Since $R$ has the same eigenvalues as $S$ it will suffice to prove Theorem 2 with $R$ in place of $S$. The following lemma uses the method of computing integral kernels introduced in [4]. 
Lemma 2. For $\varepsilon>0, \operatorname{Im} \mu>0$ and $n \neq 0, K_{n}(\varepsilon, \mu) \in \mathscr{I}_{4}$ and

$$
\left\|K_{n}(\varepsilon, \mu)\right\|_{4}^{4} \leqq \frac{\pi}{4|n| \varepsilon(\operatorname{Im} \mu)^{2}} .
$$

Proof. Let $\|\cdot\|_{p}$ denote the $\mathscr{I}_{p}$ norm. Then $\|K\|_{4}^{4}=\left\|K^{*} K\right\|_{2}^{2}$, so it suffices to show that $K^{*} K$ is Hilbert-Schmidt for $K=K_{n}(\varepsilon, \mu)$. Since (see [6], $p$ 496) $\exp \left(-i t p^{2}\right)$ has integral kernel

$$
\exp \left(-i t p^{2}\right)(x, y)=(4 \pi i t)^{-1 / 2} \exp \left(i(x-y)^{2} / 4 t\right)
$$

the integral kernel for $K=K_{n}(\varepsilon, \mu)$ is

$$
\begin{aligned}
K(x, y)= & \left(\frac{4 \pi i n}{\varepsilon}\right)^{-1 / 2} e^{-i n^{3} / 12 \varepsilon}[\varepsilon x-\mu]^{-1 / 2} e^{i(n / 2) x} \\
& \cdot \exp \left(i \varepsilon(x-y)^{2} / 4 n\right) e^{i(n / 2) y}[\varepsilon y-\mu]^{-1 / 2}
\end{aligned}
$$

Thus $K^{*} K$ has integral kernel

$$
\begin{aligned}
K^{*} K(x, y)= & \int_{-\infty}^{\infty} \overline{K(u, x)} K(u, y) d u=\left|\frac{\varepsilon}{4 \pi n}\right| \overline{[\varepsilon x-\mu]^{-1 / 2}}[\varepsilon y-\mu]^{-1 / 2} \\
& \cdot \exp \left(i\left(\frac{\varepsilon}{4 n}\left(y^{2}-x^{2}\right)+\frac{n}{2}(y-x)\right)\right) \\
& \cdot \int_{-\infty}^{\infty}|\varepsilon x-\mu|^{-1} \exp \left(i \frac{\varepsilon}{2 n}(x-y) u\right) d u .
\end{aligned}
$$

Now set $f(x)=|\varepsilon x-\mu|^{-1}$ and let $\hat{f}$ denote the Fourier transform of $f$. Then

$$
\begin{aligned}
\left\|K^{*} K\right\|_{2}^{2} & =\frac{\varepsilon^{2}}{16 \pi^{2} n^{2}} \cdot 2 \pi \int_{-\infty}^{\infty} \int_{-\infty}^{\infty} f(x) \hat{f}\left(\frac{\varepsilon}{2 n}(x-y)\right) \hat{f}\left(\frac{\varepsilon}{2 n}(y-x)\right) d x d y \\
& \leqq \frac{\varepsilon^{2}}{8 \pi n^{2}} \int_{-\infty}^{\infty} \int_{-\infty}^{\infty}|f(x)|^{2}\left|\hat{f}\left(\frac{\varepsilon}{2 n}(x-y)\right)\right|^{2} d x d y=\frac{\varepsilon}{4 \pi|n|}\|f\|^{4} .
\end{aligned}
$$

Since $\|f\|^{2}=\pi / \varepsilon \operatorname{Im} \mu$, this concludes the proof of Lemma 2 .

The next lemma plays a technical role in our proof.

Lemma 3. Let $m \geqq 4$ and let $n_{1} \ldots, n_{m}$ be non-zero integers. Let $K_{n_{j}}=K_{n_{j}}(\varepsilon, \mu)$ for $\varepsilon>0$ and $\operatorname{Im} \mu>0$ be given by (3.2). Define $\sigma=\sum_{j=1}^{n} n_{j}$.

Then

$$
\operatorname{tr}\left(\prod_{j=1}^{m} K_{n_{j}}\right)=\left[\begin{array}{cc}
0 & \text { if } \sigma \leqq 0 \\
\frac{2 \pi(i)^{m}}{(4 \pi i)^{1 / 2}} \varepsilon^{-m+1 / 2} & \sigma^{m-3 / 2} \exp \left(i \frac{\sigma \mu}{\varepsilon}\right) \\
\cdot \exp \left(-\frac{i}{12 \varepsilon} \sum_{j=1}^{m} n_{j}^{3}\right) \int_{\Omega_{m}} \exp \left(-\frac{i}{\varepsilon} C\left(m ; n_{j} ; u_{j}\right)\right) d u_{1}, \ldots, d u_{m-1} i f \sigma \geqq 0,
\end{array}\right.
$$


where $\Omega_{m}$ is the region

$$
\Omega_{m}=\left\{\left(u_{1}, \ldots, u_{m-1}\right): u_{i} \geqq 0 \text { for } i=1, \ldots, m-1 \text { and } \sum_{i=1}^{m-1} u_{i} \leqq 1\right\},
$$

$\alpha_{k}$ and $\beta_{k}$ are given by (3.6) below, and

$$
C\left(m ; n_{j} ; u_{j}\right)=\sigma^{2} \sum_{k=1}^{m} n_{k}\left(-\frac{1}{2} \beta_{k}+\frac{1}{2} \alpha_{k}+\sum_{j=1}^{k-1} \beta_{j} u_{j}-\sum_{j=k}^{m-1} \alpha_{j} u_{j}\right)^{2}
$$

Proof. From the definition of $K_{n_{j}}$, it follows that

$$
\begin{aligned}
\prod_{j=1}^{m} K_{n_{j}}= & \exp \left(-\frac{i}{12 \varepsilon} \sum_{j=1}^{m} n_{j}^{3}\right)[\varepsilon x-\mu]^{-1 / 2} \prod_{j=1}^{m-1}\left[e^{i\left(n_{j} / 2\right) x}\right. \\
& \left.\cdot \exp \left(-\frac{i}{\varepsilon} n_{j} p^{2}\right) e^{i\left(n_{j} / 2\right) x}(\varepsilon x-\mu)^{-1}\right] \\
& \cdot e^{i\left(n_{m} / 2\right) x} \exp \left(-\frac{i}{\varepsilon} n_{m} p^{2}\right) e^{i\left(n_{m} / 2\right) x}[\varepsilon x-\mu]^{-1 / 2}
\end{aligned}
$$

Using the representation

$$
(\varepsilon x-\mu)^{-1}=i \varepsilon^{-1} \int_{0}^{\infty} e^{-i x \xi} e^{i(\mu \xi / \varepsilon)} d \xi
$$

and then repeatedly applying formula (3.1) we obtain

$$
\begin{aligned}
\prod_{j=1}^{m} K_{n_{j}}= & (i)^{m-1} \varepsilon^{-m+1} \exp \left(-\frac{i}{\varepsilon} \sum_{j=1}^{m} n_{j}^{3} / 12\right)[\varepsilon x-\mu]^{-1 / 2} \\
& \cdot \int_{0}^{\infty} \ldots \int_{0}^{\infty} \exp \left(i \frac{\mu}{\varepsilon} \sum_{j=1}^{m-1} \xi_{j}\right) \exp \left(i\left(\sum_{j=1}^{m} \frac{n_{j}}{2}-\sum_{j-1}^{m-1} \alpha_{j} \xi_{j}\right) x\right) \\
& \cdot \exp \left(-\frac{i}{\varepsilon}\left\{n _ { 1 } \left(p+0+\frac{n_{2}}{2}+\frac{n_{3}}{2} \cdots+\frac{n_{m-1}}{2}+\frac{n_{m}}{2}-\alpha_{1} \xi_{1}\right.\right.\right. \\
& \left.-\alpha_{2} \xi_{2}-\cdots-\alpha_{m-1} \xi_{m-1}\right)^{2} \\
& +n_{2}\left(p-\frac{n_{1}}{2}+0+\frac{n_{3}}{2}+\cdots+\frac{n_{m-1}}{2}+\frac{n_{m}}{2}+\beta_{1} \xi_{1}-\alpha_{2} \xi_{2}\right. \\
& \left.\quad-\cdots-\alpha_{m-1} \xi_{m-1}\right)^{2}
\end{aligned}
$$

$$
+\cdots
$$




$$
\begin{aligned}
& +n_{m}\left(p-\frac{n_{1}}{2}-\frac{n_{2}}{2}-\frac{n_{3}}{2}-\cdots-\frac{n_{m-1}}{2}+0\right. \\
& \left.\left.\left.+\beta_{1} \xi_{1}+\beta_{2} \xi_{2}+\cdots+\beta_{m-1} \xi_{m-1}\right)^{2}\right\}\right) \\
& \cdot \exp \left(i\left(\sum_{j=1}^{n} \frac{n_{j}}{2}-\sum_{j=1}^{m-1} \beta_{j} \xi_{j}\right) x\right) d \xi_{1} \ldots d \xi_{m-1}[\varepsilon x-\mu]^{-1 / 2}
\end{aligned}
$$

where $\alpha_{k}, \beta_{k}$ are any pairs of real numbers satisfying $\alpha_{k}+\beta_{k}=1$ for each $k$. The expression inside the braces is quadratic in $p$. It can be written $A p^{2}+B p+C$ with $A=\sigma$. Now assume $\sigma \neq 0$ and let

$$
\begin{aligned}
& \alpha_{k}=\sigma^{-1} \sum_{i=k+1}^{m} n_{i}, \\
& \beta_{k}=\sigma^{-1} \sum_{i=1}^{k} n_{i} .
\end{aligned}
$$

With this choice of $\alpha_{k}$ and $\beta_{k}, B=0$ and

$$
C=\sum_{k=1}^{m} n_{k}\left(-\frac{\sigma}{2} \beta_{k-1}+\frac{\sigma}{2} \alpha_{k}+\sum_{j=1}^{k-1} \beta_{j} \xi_{j}-\sum_{j=k}^{m-1} \alpha_{j} \xi_{j}\right)^{2}
$$

where all undefined sums, e.g. $\alpha_{m}$ and $\beta_{0}$, are equal to zero.

Using (3.4) and (3.5) one can compute an integral kernel for $\Pi K_{n_{j}}$. Since the resultant kernel is continuous, the trace of $\Pi K_{n_{j}}$ is equal to the integral along the diagonal of this kernel. Thus

$$
\begin{aligned}
\operatorname{tr}\left(\prod_{j=1}^{m} K_{n_{j}}\right)= & (i)^{m-1} \varepsilon^{m+1}\left(\frac{4 \pi i \sigma}{\varepsilon}\right)^{-1 / 2} \exp \left(-\frac{i}{12 \varepsilon} \sum_{j=1}^{m} n_{j}^{3}\right) \\
& \cdot \int_{-\infty}^{\infty}(\varepsilon x-\mu)^{-1} \int_{0}^{\infty} \cdots \int_{0}^{\infty} \exp \left(i \frac{\mu}{\varepsilon} \sum_{j=1}^{m-1} \xi_{j}\right) \\
& \cdot \exp \left(i\left(\sigma-\sum_{j=1}^{m-1} \xi_{j}\right) x\right) \exp \left(-\frac{i}{\varepsilon} C\right) d \xi_{1} \cdots d \xi_{m-1} d x
\end{aligned}
$$

We split the integral over $\xi_{1} \cdots \xi_{m-1}$ into two parts. Let

$$
\begin{aligned}
& F_{1}(x)=\int \underset{\substack{\xi_{j} \geqq 0 \\
\Sigma \xi_{j} \leqq \sigma}}{\cdots} \int I\left(\xi_{i}, x\right) d \xi_{1} \cdots d \xi_{m-1}, \\
& F_{2}(x)=\int \underset{\substack{\xi_{j} \geqq 0 \\
\Sigma \xi_{j} \geqq \sigma}}{\cdots} \int I\left(\xi_{i}, x\right) d \xi_{1} \cdots d \xi_{m-1},
\end{aligned}
$$

where $I\left(\xi_{i}, x\right)$ is the integrand from (3.7), i.e.

$$
I\left(\xi_{i}, x\right)=\exp \left(i \frac{\mu}{\varepsilon} \sum_{j=1}^{m-1} \xi_{j}\right) \exp \left(i\left(\sigma-\sum_{j=1}^{m-1} \xi_{j}\right) x\right) \exp \left(-\frac{i}{\varepsilon} C\right) .
$$

We claim that

$$
\int_{-\infty}^{\infty}(\varepsilon x-\mu)^{-1} F_{2}(x) d x=0
$$


First, note that $F_{2}(x)$ has an analytic extension to the half plane, $\operatorname{Im} x<\operatorname{Im}(\mu / \varepsilon)$. Since $\operatorname{Im} \mu>0,(3.10)$ will follow by Cauchy's theorem if we can show that

$$
\left|F_{2}(x)\right| \leqq C|x|^{-1}
$$

for large $|x|$ in the lower half-plane. If $\sigma<0$, then

$$
\left|F_{2}(x)\right| \leqq \int \underset{\xi_{j} \geqq 0}{\cdots} \int\left|I\left(\xi_{j} ; x\right)\right| d \xi_{1} \cdots d \xi_{m-1}=e^{-\sigma \operatorname{Imx}}\left(\operatorname{Im}\left(\frac{\mu}{\varepsilon}-x\right)\right)^{-m+1}
$$

and (3.10) follows trivially.

We now consider the case $\sigma>0$. In (3.9) the change of variables $\xi_{j}=\rho \omega_{j}$ for $1 \leqq j \leqq m-2$ and $\xi_{m-1}=\rho\left(1-\sum_{j=1}^{m-2} \omega_{j}\right)$ yields

$$
F_{2}(x)=\int_{\sigma}^{\infty} f(\sigma) \exp (i \sigma x) \exp \left(i\left(\frac{\mu}{\varepsilon}-x\right) \rho\right) d \rho,
$$

where

$$
f(\rho)=\rho^{m-2} \int \underset{\substack{\omega_{j} \geqq 0 \\ \Sigma \omega_{j} \leqq 1}}{\cdots} \int \exp \left(-\frac{i}{\varepsilon} C\right) d \omega_{1} \cdots d \omega_{m-2} .
$$

In this formula $C$ is a quadratic polynomial in $\rho$. Thus,

$$
\left|f^{\prime}(\rho)\right| \leqq C_{1}+C_{2} \rho^{m-1} .
$$

Now integrate the right side of $(3.12)$ by parts. This gives

$$
F_{2}(x)=-\frac{f(\sigma) \exp (i \sigma \mu / \varepsilon)}{i(\mu / \varepsilon-x)}-\frac{\exp (i \sigma x)}{i(\mu / \varepsilon-x)} \int_{\sigma}^{\infty} f^{\prime}(\rho) \exp (i(\mu / \varepsilon-x) \rho) d \rho .
$$

Since for $\operatorname{Im} x<0$,

$$
\begin{aligned}
& \left|\exp (i \sigma x) \int_{\sigma}^{\infty} f^{\prime}(\rho) \exp (i(\mu / \varepsilon-x) \rho) d \rho\right| \\
& \quad \leqq \exp (-\sigma \operatorname{Im} x) \int_{\sigma}^{\infty}\left(C_{1}+C_{2} \rho^{m-1}\right) \exp ((-\operatorname{Im}(\mu / \varepsilon)+\operatorname{Im} x) \rho) d \rho \\
& \quad \leqq C
\end{aligned}
$$

Eq. (3.13) implies (3.11). This proves (3.10) when $\sigma \neq 0$. It also establishes Lemma 3 for the case $\sigma<0$, since, if $\sigma<0$, then $F_{1}(x)=0$. To handle the case $\sigma>0$ note that $F_{1}(x)$ extends to be entire in $x$. By an analysis similar to the one above we obtain

$$
F_{1}(x)=\frac{f(\sigma) \exp (i \sigma \mu / \varepsilon)}{i(\mu / \varepsilon-x)}-\frac{\exp (i \sigma x)}{i(\mu / \varepsilon-x)} \int_{0}^{\sigma} f^{\prime}(\rho) \exp (i(\mu / \varepsilon-x) \rho) d \rho .
$$


Since for $\operatorname{Im} x>0$,

$$
\begin{aligned}
& \left|\exp (i \sigma x) \int_{0}^{\sigma} f^{\prime}(\rho) \exp (i(\mu / \varepsilon-x) \rho) d \rho\right| \\
& \quad \leqq \exp (-\sigma \operatorname{Im} x)\left(C_{1}+C_{2} \sigma^{m-1}\right) \int_{0}^{\sigma} \exp ((-\operatorname{Im} \mu / \varepsilon+\operatorname{Im} x) \rho) d \rho \\
& \quad \leqq C
\end{aligned}
$$

it follows that $F_{1}(x)$ decreases rapidly enough in the upper half-plane to allow the evaluation of the integral of $(\varepsilon x-\mu)^{-1} F_{1}(x)$ by a contour in the upper half-plane. Since $(\varepsilon x-\mu)^{-1}$ has a simple pole there, this gives

$$
\int_{-\infty}^{\infty}(\varepsilon x-\mu)^{-1} F_{1}(x) d x=2 \pi i \varepsilon^{-1} F_{1}(\mu / \varepsilon)
$$

Using (3.7) through (3.10) and making the change of variables $\sigma u_{i}=\xi_{i}$ concludes the proof of Lemma 3 for the case $\sigma>0$.

The case $\sigma=0$ requires a separate argument. We omit the details, noting that the precise value of the trace when $\sigma=0$ is not crucial in what follows.

From Lemma 2 it follows that for $\varepsilon>0, \operatorname{Im} \mu>0$ and $z \neq 0, R(\varepsilon, \mu, z) \in \mathscr{I}_{4}$. Thus $\operatorname{det}_{4}(1+R)$ is well defined. It follows easily from (3.2) that

$$
\|R(\varepsilon, \mu, z)\| \leqq C\left(|z|^{N}+|z|^{-N}\right)(\operatorname{Im} \mu)^{-1} .
$$

Now choose $M_{0}>0$ and a neighbourhood $\mathscr{N}$ of $|z|=1$, and that for $\operatorname{Im} \mu>M_{0}$ and $z \in \mathscr{N},\|R(\varepsilon, \mu, z)\|<1$. Then for such $\mu$ and $z, \ln _{\operatorname{det}_{4}}(1+R(\varepsilon, \mu, z))$ is well defined and analytic in $z$.

Lemma 4. If $\varepsilon>0$ and $\operatorname{Im} \mu>M_{0}$, then $\ln \operatorname{det}_{4}(1+R(\varepsilon, \mu, z))$ extends to be analytic in $|z|$ for $|z|<1$.

Proof. If $\operatorname{Im} \mu>M_{0}$ and $z \in \mathscr{N}$ then $\|R(\varepsilon, \mu, z)\|<1$. Thus the eigenvalues $\left\{\lambda_{k}\right\}$ of $R$ must satisfy $\sup _{k}\left|\lambda_{k}\right|<1$. Using this fact and Theorem 9.2(a) from [8], it is not difficult to see that

$$
\ln \operatorname{det}_{4}(1+R(\varepsilon, \mu, z))=\sum_{m=4}^{\infty} \frac{(-1)^{m+1}}{m} \operatorname{tr}\left(R(\varepsilon, \mu, z)^{m}\right),
$$

where the convergence is uniform for $z \in \mathcal{N}$.

By Lemma $3, \operatorname{tr}\left(R(\varepsilon, \mu, z)^{m}\right)$ extends to be analytic on $|z|<1$, so the conclusion of Lemma 2 follows by the maximum modulus principle.

For the remainder of this section fix $\mu_{0}$ with $\operatorname{Im} \mu_{0}>M_{0}$. Define the functions $f_{\ell}(\varepsilon)$ for $\varepsilon>0$ by

$$
\ln \operatorname{det}_{4}\left(1+R\left(\varepsilon, \mu_{0}, z\right)\right)=\sum_{\ell=1}^{\infty} f_{\ell}(\varepsilon) z^{\ell}
$$

for $|z|<1$. Let $\mathscr{E}$ denote the set of $\varepsilon$ for which $R\left(\varepsilon, \mu_{0}, z\right)$ never has eigenvalue -1 as $z$ ranges through $\mathbb{C} \backslash\{0\}$. 
Let $N_{1}$ be the largest integer such that $c_{N_{1}} \neq 0$, where $c_{n}$ denotes the coefficient in $q(x)$.

Lemma 5. If $\varepsilon \in \mathscr{E}$ then $\ln \operatorname{det}_{4}(1+R(\varepsilon, \mu, z))$ is a polynomial in $z$ of degree at most $4 N_{1}$, i.e. $f_{\ell}(\varepsilon)=0$ for $\ell>4 N_{1}$.

Proof. If $\varepsilon \in \mathscr{E}$ then $\ln \operatorname{det}_{4}\left(1+R\left(\varepsilon, \mu_{0}, z\right)\right)$ extends to be entire in $z$. Using Lemma 2 and Theorem 9.2 (b) from [8] we see that

$$
\begin{aligned}
& \left|\ln \operatorname{det}_{4}\left(1+R\left(\varepsilon, \mu_{0}, z\right)\right)\right| \leqq C_{1}\left\|R\left(\varepsilon, \mu_{0}, z\right)\right\|_{4}^{4} \\
& \leqq C_{2}\left(|z|^{4 N_{1}}+|z|^{-4 N}\right) \varepsilon^{-1}\left(\operatorname{Im} \mu_{0}\right)^{-2} .
\end{aligned}
$$

These two facts imply the conclusion of Lemma 5.

Lemma 5 shows that $\mathscr{E}$ must lie in the zero set of $f_{\ell}(\varepsilon)$ for every $\ell>4 N_{1}$. Thus the following lemma will complete the proof of Theorem 2.

Lemma 6. The function $f_{5 N_{1}}(\varepsilon)$ satisfies

$$
\lim _{\varepsilon \rightarrow \infty} \varepsilon^{5-1 / 2} f_{5 N_{1}}(\varepsilon) \neq 0 .
$$

Proof. From (3.2), (3.14), (3.15) and Lemma 3 it follows that

$$
\begin{aligned}
& \varepsilon^{5-1 / 2} f_{5 N_{1}}(\varepsilon)=\varepsilon^{5-1 / 2} \sum_{m=4}^{\infty} \sum_{\substack{N \leq n_{1}, . . n_{m} \leqq N_{1} \\
\bar{\Sigma} n_{j}=5 N_{1}}} \frac{(-1)^{m+1}}{m} \\
& \cdot c_{n_{1}} \cdots c_{n_{m}} \operatorname{tr}\left(K_{n_{1}} \cdots K_{n_{m}}\right) \\
& =\sum_{m=5}^{\infty} \sum_{\substack{\text { s. } \\
-N n_{1}, \ldots, n_{m} \leqq N_{1} \\
\Sigma n_{j}=5 N_{1}}} \frac{(-1)^{m+1}}{m} \\
& \cdot c_{n_{1}} \cdots c_{n_{m}} \frac{2 \pi(i)^{m}}{(4 \pi i)^{1 / 2}} \varepsilon^{5-m}\left(5 N_{1}\right)^{m-3 / 2} \exp \left(i 5 N_{1} \mu_{0} / \varepsilon\right) \\
& \cdot \exp -\left(\frac{i}{12 \varepsilon} \sum_{j=1}^{m} n_{j}\right) \int_{\Omega_{m}} \exp \left(-\frac{i}{\varepsilon} C\left(m ; n_{i} ; u_{i}\right)\right) d u_{1} \ldots d u_{m-1} \text {. }
\end{aligned}
$$

The interchange of sums used to derive this formula can be justified by noting that the series obtained from the substitution of (3.2) in (3.14) converges absolutely for small $|z|$. To obtain the relevant estimate, use Lemma 3 to express $\operatorname{tr}\left(K_{n_{1}} \cdots K_{n_{m}}\right)$ and the fact that $\left|\Omega_{m}\right|=\left((m-1) !^{-1}\right.$. We also used the fact that if $\sum_{j=1}^{m} n_{j}=5 N_{1}$ with $n_{j}<N_{1}$, then $m$ must be at least 5 . The series in $m$ can be bounded by a fixed $\ell_{1}$ sequence for $\varepsilon$ large. Thus, we can take the limit inside the sum. Only the $m=5$ term survives:

$$
\lim _{\varepsilon \rightarrow \infty} \varepsilon^{5-1 / 2} f(\varepsilon)=\frac{(-1)^{6}}{5} c_{N_{1}}^{5} \frac{2 \pi(i)^{5}}{(4 \pi i)^{1 / 2}}\left(5 N_{1}\right)^{5-3 / 2}(4 !)^{-1} \neq 0 .
$$

This establishes Lemma 6. 
Acknowledgement. It is a pleasure to thank I. Herbst and J. Howland for suggesting this problem and Barry Simon for a useful conversation.

\section{References}

1. Avron, J. E.: Model calculation of Stark ladder resonances. Phys. Rev. Lett. 37, 1568-1571 (1976)

2. Arvon, J. E.: On the spectrum of $p^{2}+V(x)+\varepsilon x$, with $V$ periodic and $\varepsilon$ complex. J. Phys. A. 12, 2393 (1979)

3. Avron, J. E., Grossmann, A., Gunther, L., Zak, J.: Instability of the continuous spectrum: The $N$-band Stark ladders. J. Math. Phys. 18, 918 (1977)

4. Bentosela, F., Carmona, R., Duclos, P., Simon, B., Soulliard, B., Weder, R.: Commun. Math. Phys. 88, 387-397 (1983)

5. Herbst, I. W., Howland, J. S.: Commun. Math. Phys. 80, 23-42 (1981)

6. Kato, T.: Perturbation theory for linear operators. Berlin, Heidelberg, New York: Springer 1980

7. Nenciu, A., Nenciu, G.: E17-82-207, E17-82-208, JINR, Dubna, 1982

8. Simon, B.: Trace ideals and their applications. Cambridge: Cambridge University Press 1979

9. Wannier, G. H.: Wave functions and effective Hamiltonian for Bloch electrons in an electric field. Phys. Rev. 117, 432 (1960)

10. Wannier, G. H.: Stark ladder in solids? A reply. Phys. Rev. 181, 1364 (1969)

11. Zak, J.: Stark ladder in solids; A reply to reply. Phys. Rev. 181, 1360 (1969)

Communicated by B. Simon

Received November 2, 1984; in revised form January 14, 1985 
